\title{
Possible scenarios for single, double, or multiple kinetic freeze-out in high energy collisions
}

\author{
Muhammad Waqas ${ }^{1}, \mathrm{Fu}-\mathrm{Hu} \mathrm{Liu}^{1}$ 田, Sakina Fakhraddin²,3, Magda A. Rahim²,3 \\ ${ }^{1}$ Institute of Theoretical Physics \& State Key Laboratory of Quantum Optics and Quantum Optics Devices, \\ Shanxi University, Taiyuan, Shanxi 030006, China \\ ${ }^{2}$ Physics Department, College of Science $\mathcal{E}$ Arts in Riyadh Al-Khabra, \\ Qassim University, Qassim, Kingdom of Saudi Arabia \\ ${ }^{3}$ Physics Department, Faculty of Science, Sana'a University, P.O. Box 1247, Sana'a, Republic of Yemen
}

\begin{abstract}
Transverse momentum spectra of different types of particles produced in mid-rapidity interval in central and peripheral gold-gold $(\mathrm{Au}-\mathrm{Au})$ collisions, central and peripheral deuteron-gold $(\mathrm{d}-\mathrm{Au})$ collisions, and inelastic (INEL) or non-single-diffractive (NSD) proton-proton $(p p)$ collisions at the Relativistic Heavy Ion Collider (RHIC), as well as in central and peripheral lead-lead (Pb-Pb) collisions, central and peripheral proton-lead $(p-\mathrm{Pb})$ collisions, and INEL or NSD $p p$ collisions at the Large Hadron Collider (LHC) are analyzed by the blastwave model with Boltzmann-Gibbs statistics. The model results are largely consist with the experimental data in special transverse momentum ranges measured by the PHENIX, STAR, ALICE, and CMS Collaborations. It is showed that the kinetic freeze-out temperature of emission source is dependent on particle mass, which reveals the scenario for multiple kinetic freeze-out in collisions at the RHIC and LHC. The scenario for single or double kinetic freeze-out is not observed in this study.
\end{abstract}

Keywords: kinetic freeze-out temperature, scenario for multiple kinetic freeze-out, high energy collisions PACS: $25.75 . \mathrm{Ag}, 25.75 . \mathrm{Dw}, 24.10 . \mathrm{Pa}$

\section{Introduction}

Chemical freeze-out is an important intermediate stage in high energy collisions. During this stage, the intra-nuclear collisions among particles are inelastic, and ratios of different types of particles remain invariant. Chemical freeze-out temperature $\left(T_{c h}\right)$ is an important quantity which describes the excitation degree of interacting system at the stage of chemical freeze-out. It is important to know about the various $T_{c h}$ at the stage of chemical freeze-out. Correspondingly, kinetic freezeout is the last stage, but not least one, in high energy collisions, in which the intra-nuclear collisions among particles are elastic and the transverse momentum distributions of different types of particles are no longer changed. Kinetic freeze-out temperature $\left(T_{0}\right.$ or $\left.T_{k i n}\right)$ is also an important quantity which describes the excitation degree of the interacting system at the stage of kinetic freeze-out. A natural question is that how many different $T_{0}$ are there at the stage of kinetic freeze-out.

Generally, the kinetic freeze-out happens later than or simultaneously with the chemical freeze-out. This renders that $T_{0}$ is smaller than or equal to $T_{c h}$. According to the thermal and statistical model [1-4], with the increase of the collision energy, the single $T_{c h}$ in central nucleus-nucleus collisions increases from a few $\mathrm{GeV}$ to above $10 \mathrm{GeV}$, and then saturates in an energy range more than dozens of $\mathrm{GeV}$. The maximum $T_{c h}$ at the top Relativistic Heavy Ion Collider (RHIC) and the Large Hadron Collider (LHC) energies is about $160 \mathrm{MeV}$, though there is a slightly increase from the top RHIC energy to that of LHC. Meanwhile, $T_{c h}$ in central nucleus-nucleus collisions is slightly larger than that in peripheral nucleus-nucleus collisions. The properties of $T_{c h}$ is well acknowledged in the community.

However, the situation of $T_{0}$ is more complex. Firstly, with increasing the collision energy, although $T_{0}$ in central collisions increases initially over the energy range from a few $\mathrm{GeV}$ to above $10 \mathrm{GeV}$, the subsequent tendency can be saturated, increscent, or decrescent. It is interesting to know the correct subsequent tendency. Secondly, $T_{0}$ in central collisions can be larger than, equal to, or smaller than that in peripheral collisions. It is important to figure out which $T_{0}$ is larger. Thirdly, $T_{0}$ can possibly give single, double, or multiple values for the emissions of different types of particles in the given collisions. Which freeze-out scenario is correct also interests us.

It is crucial but also challenging to solve all the above three issues. In particular, the first issue needs to study

*E-mail: fuhuliu@163.com; fuhuliu@sxu.edu.cn 
the excitation function of $T_{0}$, which needs the collection and analysis of many experimental data. We shall do it in other work. The second issue is studied in our very recent work [5] which shows a slightly larger $T_{0}$ in central collisions if a non-zero transverse flow velocity $\left(\beta_{T}\right)$ is used for peripheral collisions. Considering the low multiplicity of the final state particles, peripheral collisions at the RHIC are similar to inelastic (INEL) or nonsingle-diffractive (NSD) proton-proton $(p p)$ collisions at the LHC. Given the low frequency of the cascade collisions in both participant nucleons, peripheral collisions are also similar to INEL or NSD $p p$ collisions. In fact, $p p$ collisions also show collective expansion [6] and then reveal non-zero $\beta_{T}$. For the third issue, we have to extract $T_{0}$ from the transverse momentum $\left(p_{T}\right)$ spectra of different types of particles. As an accompanying result, $\beta_{T}$ can be obtained, which also shows complex situation as $T_{0}$.

Several methods can be used to calculate $T_{0}$ and $\beta_{T}$. In the present work, we shall use the blast-wave model with Boltzmann-Gibbs statistics [7-9] to extract $T_{0}$ and $\beta_{T}$ from $p_{T}$ spectra of different types of particles produced in mid-rapidity interval in central and peripheral gold-gold $(\mathrm{Au}-\mathrm{Au})$ collisions, central and peripheral deuteron-gold $(d-\mathrm{Au})$ collisions, and INEL or NSD $p p$ collisions at the RHIC, as well as in central and peripheral lead-lead $(\mathrm{Pb}-\mathrm{Pb})$ collisions, central and peripheral proton-lead $(p$-Pb) collisions, and INEL or NSD $p p$ collisions at the LHC. The contribution of soft excitation process is included, while the contribution of hard scattering process is not excluded, if available in low $p_{T}$ region. The model results are compared with the experimental data measured by the PHENIX [10-14], STAR [15-18], ALICE [19-27], and CMS [28] Collaborations.

The remainder of this paper is structured as follows. The method and formalism are shortly described in Section 2. Results and discussions are given in Section 3. In Section 4, we summarize our main observations and conclusions.

\section{The method and formalism}

A few methods can be used to extract $T_{0}$ and $\beta_{T}$, including but not limited to, i) the blast-wave model with Boltzmann-Gibbs statistics [7-9], ii) the blast-wave model with Tsallis statistics [29], iii) an alternative method using the Boltzmann distribution [8, 30-36], iv) the alternative method using the Tsallis distribution [37, 38]. It should be noted that, in the alternative method, $T_{0}$ is the intercept in the linear relation $T-m_{0}$, where $T$ is the effective temperature which includes the contributions of thermal motion and flow effect, and $m_{0}$ is the rest mass; and $\beta_{T}$ is the slope in the linear relation $\left\langle p_{T}\right\rangle-\bar{m}$, where $\left\langle p_{T}\right\rangle$ is the mean transverse momentum and $\bar{m}$ is the mean moving mass (i.e. the mean energy).
Our very recent work [5] confirms that the above methods are harmonious. Among these methods, the first one is the most direct and has fewer parameters, though it is revised in different ways and applied to other quantities [39-43]. In the present work, we have used the first method i.e. the blast-wave model with BoltzmannGibbs statistics, to extract $T_{0}$ and $\beta_{T}$. Other methods will not be used due to their coherence [5]. As an application of the blast-wave model with Boltzmann-Gibbs statistics, we shall only give a short representation of its formalism in the original form. The discussions on its various revisions and applications to derive other quantities are beyond the focus of the present work. We will not discuss them further.

According to refs. [7-9], the blast-wave model with Boltzmann-Gibbs statistics gives the following $p_{T}$ distribution

$$
\begin{aligned}
f_{1}\left(p_{T}\right)= & \frac{1}{N} \frac{d N}{d p_{T}}=C p_{T} m_{T} \int_{0}^{R} r d r \\
& \times I_{0}\left[\frac{p_{T} \sinh (\rho)}{T_{0}}\right] K_{1}\left[\frac{m_{T} \cosh (\rho)}{T_{0}}\right],
\end{aligned}
$$

where $N$ is the number of particles, $C$ is the normalized constant, $m_{T}=\sqrt{p_{T}^{2}+m_{0}^{2}}$ is the transverse mass, $I_{0}$ and $K_{1}$ are the modified Bessel functions of the first and second kinds respectively, $\rho=\tanh ^{-1}[\beta(r)]$ is the boost angle, $\beta(r)=\beta_{S}(r / R)^{n_{0}}$ is a self-similar flow profile, $\beta_{S}$ is the flow velocity on the surface, $r / R$ is the relative radial position in the thermal source, and $n_{0}$ is a free parameter [7].

One has the relation, $\beta_{T}=\left(2 / R^{2}\right) \int_{0}^{R} r \beta(r) d r=$ $2 \beta_{S} /\left(n_{0}+2\right)$, between $\beta_{T}$ and $\beta(r)$. In the present work, we use $n_{0}=2$ from ref. [7], which results in $\beta_{T}=0.5 \beta_{S}$. Because the maximum value of $\beta_{S}$ is $1 c$, the maximum value of $\beta_{T}$ is $0.5 c$. In other work [29] which concerns the blast-wave model with Tsallis statistics, $n_{0}=1$, which results in $\beta_{T}=(2 / 3) \beta_{S}$. Thus, the maximum $\beta_{T}$ is $(2 / 3)$ c. Another work [19] uses $n_{0}$ to be a non-integer from that less than 1 to above 2 , which corresponds to the centrality from center to periphery. This can lead to a large variation in $\beta_{T}$. As a not too sensitive quantity, the selection of $n_{0}$ is flexible. Although different $n_{0}$ can be used to fit $p_{T}$ spectrum, it impacts generally $T_{0}$. Meanwhile, $\beta_{T}$ also impacts $T_{0}$.

Generally, there are mainly two processes, the soft excitation process and the hard scattering process, in the contributions for $p_{T}$ spectrum. The soft excitation process contributes $p_{T}$ spectrum in a narrow range. The hard excitation process contributes $p_{T}$ spectrum in a wide range. The blast-wave model with BoltzmannGibbs statistics and other methods mentioned above describe only the contribution of soft excitation process. For the contribution of hard scattering process, we can use an inverse power-law [44-46], i.e. the Hage- 
dorn function $[47,48]$

$$
f_{2}\left(p_{T}\right)=\frac{1}{N} \frac{d N}{d p_{T}}=A p_{T}\left(1+\frac{p_{T}}{p_{0}}\right)^{-n}
$$

where $p_{0}$ and $n$ are free parameters and $A$ is the normalization constant related to the free parameters. The Hagedorn function has three revisions [49-55] which will not be discussed here because they are out of the scope of this work. To describe a wide $p_{T}$ spectrum, we can use a superposition of the two contributions.

We have two methods to superpose the two functions, $f_{1}\left(p_{T}\right)$ and $f_{2}\left(p_{T}\right)$. Considering the continuities of the two functions from 0 to each maximum, we have

$$
f_{0}\left(p_{T}\right)=\frac{1}{N} \frac{d N}{d p_{T}}=k f_{1}\left(p_{T}\right)+(1-k) f_{2}\left(p_{T}\right),
$$

where $k$ denotes the contribution fraction of the first component, $f_{1}\left(p_{T}\right)$, the soft excitation process. According to Hagedorn's model [47], we can also use the usual step function

$$
\begin{aligned}
f_{0}\left(p_{T}\right) & =\frac{1}{N} \frac{d N}{d p_{T}} \\
& =A_{1} \theta\left(p_{1}-p_{T}\right) f_{1}\left(p_{T}\right)+A_{2} \theta\left(p_{T}-p_{1}\right) f_{2}\left(p_{T}\right)
\end{aligned}
$$

to superpose the two functions. Here $A_{1}$ and $A_{2}$ are constants which result in the two functions to be equal at $p_{T}=p_{1}$.

The first superposition (Eq. (3)) has been used in our recent work [5]. In a low $p_{T}$ region, there is an entanglement between $f_{1}\left(p_{T}\right)$ and $f_{2}\left(p_{T}\right)$ in the first superposition, though the contribution of $f_{1}\left(p_{T}\right)$ dominates in most cases. We will use the second superposition (Eq. $(4))$ in the present work. In the second superposition, there is no entanglement between $f_{1}\left(p_{T}\right)$ and $f_{2}\left(p_{T}\right)$ in different $p_{T}$ regions. In particular, $f_{1}\left(p_{T}\right)$ contributes only in a low $p_{T}$ region, and $f_{2}\left(p_{T}\right)$ contributes only in a high $p_{T}$ region. A narrow $p_{T}$ range such as $0-5 \mathrm{GeV} / c$ is enough to extract $T_{0}$ and $\beta_{T}$ by the second superposition. In the case of encountering a wide $p_{T}$ spectrum, we can cut only the range of $0-5 \mathrm{GeV} / c$ for analysis in the second superposition. In fact, even a range of $0-$ $2 \sim 3 \mathrm{GeV} / c$ is already wide enough in some cases. In the calculation, only $f_{1}\left(p_{T}\right)$ is used in the low $p_{T}$ region which is a special region as wide as possible, and $f_{2}\left(p_{T}\right)$ which can be used in the high $p_{T}$ region is not used, as it is being beyond the focus of the present work.

Both the superpositions in Eqs. (3) and (4) are normalized to 1 because they are the probability density functions. We need a normalization constant, $N_{0}$, in the fitted process if the experimental data are not normalized to 1 . In many cases, the experimental data presented in literature are not in the form of probability density function. Thus, we need an appropriate transformation for the superpositions so that we can compare them with the experimental data. For example, for the main three forms of experimental $p_{T}$ spectra, $\left(1 / 2 \pi p_{T}\right) d^{2} N / d p_{T} d y, d^{2} N / d p_{T} d y$, and $d N / d p_{T}$, one can use $\left(1 / 2 \pi p_{T}\right) N_{0} f_{0}\left(p_{T}\right) / d y, N_{0} f_{0}\left(p_{T}\right) / d y$, and $N_{0} f_{0}\left(p_{T}\right)$ to fit them, respectively. The purpose of the present work is to extract $T_{0}$ and $\beta_{T}$. The meaning and value of $N_{0}$ are less important, though $N_{0}$ can be obtained at the volley. Instead, the ratio of $N_{0}$ for different types of particles in the same form of spectra measured in the same experimental condition have important meanings.

\section{Results and discussion}

Figure 1 presents the transverse momentum spectra, $\left(1 / 2 \pi p_{T}\right) d^{2} N / d p_{T} d y$, of different types of particles produced in mid-rapidity interval in $\mathrm{Au}-\mathrm{Au}$ collisions at center-of-mass energy $\sqrt{s_{N N}}=200 \mathrm{GeV}$ at the RHIC. The symbols represent the experimental data measured by the PHENIX [10] and STAR [15, 16] Collaborations. The curves are our fitted results by using the blast-wave model with Boltzmann-Gibbs statistics, Eq. (1). In the bottom part of each panel, the results of data/fit are presented to monitor the difference between fit and data. The left and right panels are the results corresponding to central (0-5\% centrality) and peripheral (60-92\% or $60-80 \%$ centrality) collisions respectively. The upper, middle, and lower panels are the results corresponding to positive pions $\left(\pi^{+}\right)$, positive kaons $\left(K^{+}\right)$, and protons $(p)$; negative pions $\left(\pi^{-}\right)$, negative kaons $\left(K^{-}\right)$, and anti-protons $(\bar{p})$; as well as neutral $\phi$ mesons and negative $\Xi^{-}$baryons, respectively. In each fitting, the method of least squares is used, in which the minimum $\chi^{2}$ is used to determine various parameters, where $\chi^{2}=\sum_{i}\left[\left(\left.f_{i}\right|_{\exp }-\left.f_{i}\right|_{\text {th }}\right)^{2} / \sigma_{i}^{2}\right],\left.f_{i}\right|_{\text {exp }}$ denotes the experimental probability, $\left.f_{i}\right|_{\text {th }}$ denotes the theoretical probability, and $\sigma_{i}$ denotes the experimental error, corresponding to the $i$ th data point. The values of free parameters $\left(T_{0}\right.$ and $\left.\beta_{T}\right)$, normalization constant $\left(N_{0}\right), \chi^{2}$, and degrees of freedom (dof) are listed in Table 1 . In most cases, the confidence levels of fittings are $95 \%$. In a few cases, these values are $90 \%$. To avoid trivialness, the concrete confidence levels are not listed in the table one by one. One can see that the model results describe approximately the experimental data in special transverse momentum ranges in large collision system measured at the RHIC by the PHENIX and STAR Collaborations. The special transverse momentum ranges for some particles in peripheral $\mathrm{Au}-\mathrm{Au}$ collisions are only in $0-2 \sim 3$ $\mathrm{GeV} / c$. In other cases, the particles are expected to have wider special transverse momentum ranges.

Figure 2 is the same as Fig. 1, but it shows the transverse momentum spectra of different types of particles produced in mid-rapidity interval in $d$-Au collisions at $\sqrt{s_{N N}}=200 \mathrm{GeV}$. The symbols represent the exper- 

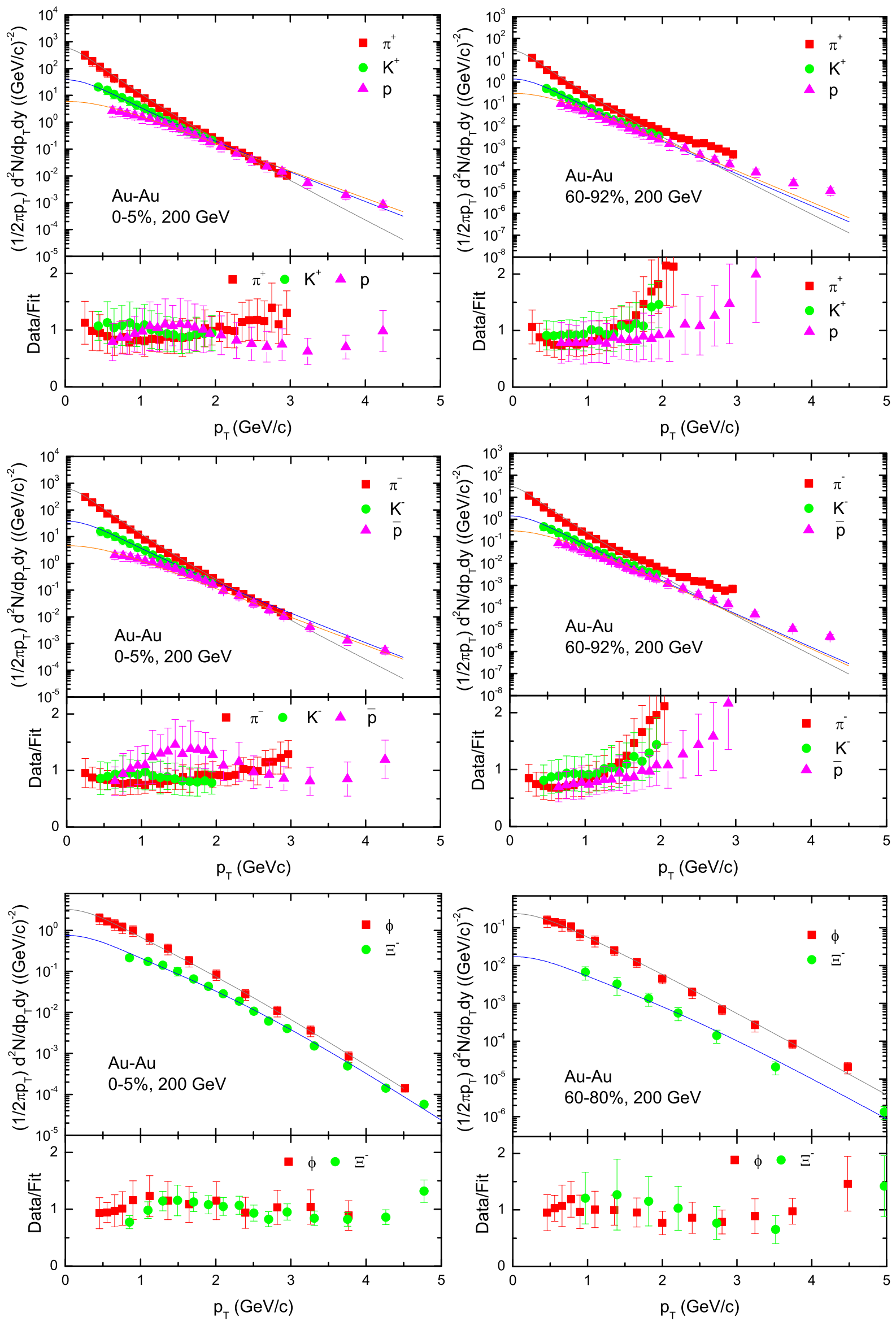

Fig. 1. Transverse momentum spectra of different types of particles produced in $\mathrm{Au}$-Au collisions at $\sqrt{s_{N N}}=200 \mathrm{GeV}$ at the RHIC. The symbols represent the experimental data measured by the PHENIX [10] and STAR [15, 16] Collaborations and the curves are our fitted results by using the blast-wave model with Boltzmann-Gibbs statistics, Eq. (1). Following each panel, the results of data/fit are presented. Left: for central collisions ( $0-5 \%$ centrality); Right: for peripheral collisions (60-92\% or $60-80 \%$ centrality). Upper: for $\pi^{+}, K^{+}$, and $p$ with $|\eta|<0.35$ [10]; Middle: for $\pi^{-}, K^{-}$, and $\bar{p}$ with $|\eta|<0.35$ [10]; Lower: for $\phi$ with $|y|<0.5$ [15] and $\Xi^{-}$with $|y|<0.75$ [16]. 
Table 1. Values of $T_{0}, \beta_{T}, N_{0}, \chi^{2}$, and dof corresponding to the curves in Figs. 1-6.

\begin{tabular}{|c|c|c|c|c|c|c|}
\hline Figure & Centrality & Particle & $T_{0}(\mathrm{GeV})$ & $\beta_{T}(c)$ & $N_{0}$ & $\chi^{2} /$ dof \\
\hline Fig. 1 & $0-5 \%$ & $\pi^{+}$ & $0.152 \pm 0.001$ & $0.344 \pm 0.001$ & $47.60 \pm 2.40$ & $13 / 25$ \\
\hline $\mathrm{Au}-\mathrm{Au}$ & & $K^{+}$ & $0.120 \pm 0.002$ & $0.440 \pm 0.003$ & $7.70 \pm 0.30$ & $6 / 13$ \\
\hline \multirow[t]{14}{*}{$200 \mathrm{GeV}$} & & $p$ & $0.220 \pm 0.002$ & $0.321 \pm 0.002$ & $2.66 \pm 0.10$ & $10 / 19$ \\
\hline & & $\pi^{-}$ & $0.140 \pm 0.001$ & $0.368 \pm 0.001$ & $49.50 \pm 2.00$ & $30 / 25$ \\
\hline & & $K^{-}$ & $0.120 \pm 0.002$ & $0.335 \pm 0.002$ & $7.70 \pm 0.25$ & $10 / 13$ \\
\hline & & $\bar{p}$ & $0.210 \pm 0.001$ & $0.333 \pm 0.002$ & $1.97 \pm 0.10$ & $17 / 19$ \\
\hline & & $\phi$ & $0.125 \pm 0.002$ & $0.400 \pm 0.002$ & $1.20 \pm 0.01$ & $2 / 11$ \\
\hline & & $\Xi^{-}$ & $0.120 \pm 0.001$ & $0.395 \pm 0.001$ & $0.56 \pm 0.03$ & $15 / 12$ \\
\hline & $60-92 \%$ & $\pi^{+}$ & $0.115 \pm 0.003$ & $0.347 \pm 0.003$ & $1.70 \pm 0.05$ & $74 / 25$ \\
\hline & & $K^{+}$ & $0.090 \pm 0.002$ & $0.400 \pm 0.003$ & $0.20 \pm 0.01$ & $11 / 13$ \\
\hline & & $p$ & $0.130 \pm 0.002$ & $0.338 \pm 0.003$ & $0.090 \pm 0.003$ & $14 / 19$ \\
\hline & & $\pi^{-}$ & $0.115 \pm 0.002$ & $0.372 \pm 0.003$ & $1.80 \pm 0.08$ & $46 / 25$ \\
\hline & & $K^{-}$ & $0.090 \pm 0.003$ & $0.407 \pm 0.004$ & $0.20 \pm 0.01$ & $4 / 13$ \\
\hline & & $\bar{p}$ & $0.138 \pm 0.003$ & $0.308 \pm 0.004$ & $0.090 \pm 0.004$ & $31 / 19$ \\
\hline & $60-80 \%$ & $\phi$ & $0.150 \pm 0.001$ & $0.380 \pm 0.001$ & $0.095 \pm 0.002$ & $4 / 11$ \\
\hline & & $\Xi^{-}$ & $0.135 \pm 0.002$ & $0.390 \pm 0.002$ & $0.014 \pm 0.002$ & $4 / 4$ \\
\hline \multirow{16}{*}{$\begin{array}{c}\text { Fig. } 2 \\
d-\mathrm{Au} \\
200 \mathrm{GeV}\end{array}$} & $0-20 \%$ & $\pi^{+}$ & $0.120 \pm 0.001$ & $0.439 \pm 0.001$ & $1.00 \pm 0.10$ & $21 / 21$ \\
\hline & & $K^{+}$ & $0.280 \pm 0.002$ & $0.200 \pm 0.002$ & $0.11 \pm 0.01$ & $12 / 18$ \\
\hline & & $p$ & $0.202 \pm 0.002$ & $0.340 \pm 0.002$ & $0.060 \pm 0.005$ & $6 / 21$ \\
\hline & & $\pi^{-}$ & $0.120 \pm 0.001$ & $0.442 \pm 0.001$ & $0.87 \pm 0.07$ & $9 / 21$ \\
\hline & & $K^{-}$ & $0.270 \pm 0.002$ & $0.204 \pm 0.005$ & $0.11 \pm 0.01$ & $2 / 18$ \\
\hline & & $\bar{p}$ & $0.208 \pm 0.002$ & $0.330 \pm 0.002$ & $0.042 \pm 0.002$ & $22 / 21$ \\
\hline & & $\eta$ & $0.125 \pm 0.001$ & $0.436 \pm 0.001$ & $0.065 \pm 0.004$ & $9 / 3$ \\
\hline & & $\phi$ & $0.110 \pm 0.002$ & $0.420 \pm 0.002$ & $0.015 \pm 0.001$ & $7 / 5$ \\
\hline & $60-88 \%$ & $\pi^{+}$ & $0.125 \pm 0.002$ & $0.388 \pm 0.002$ & $0.30 \pm 0.03$ & $5 / 21$ \\
\hline & & $K^{+}$ & $0.265 \pm 0.002$ & $0.140 \pm 0.003$ & $0.030 \pm 0.002$ & $25 / 18$ \\
\hline & & $p$ & $0.177 \pm 0.001$ & $0.290 \pm 0.002$ & $0.015 \pm 0.001$ & $25 / 21$ \\
\hline & & $\pi^{-}$ & $0.117 \pm 0.002$ & $0.400 \pm 0.002$ & $0.30 \pm 0.01$ & $21 / 21$ \\
\hline & & $K^{-}$ & $0.251 \pm 0.001$ & $0.155 \pm 0.003$ & $0.030 \pm 0.001$ & $10 / 18$ \\
\hline & & $\bar{p}$ & $0.199 \pm 0.002$ & $0.285 \pm 0.003$ & $0.010 \pm 0.001$ & $11 / 21$ \\
\hline & & $\eta$ & $0.110 \pm 0.003$ & $0.445 \pm 0.002$ & $0.018 \pm 0.002$ & $1 / 3$ \\
\hline & & $\phi$ & $0.155 \pm 0.004$ & $0.375 \pm 0.003$ & $0.0027 \pm 0.0004$ & $6 / 5$ \\
\hline \multirow{8}{*}{$\begin{array}{c}\text { Fig. } 3 \\
p p \\
200 \mathrm{GeV}\end{array}$} & - & $\pi^{+}$ & $0.114 \pm 0.002$ & $0.402 \pm 0.002$ & $3.78 \pm 0.14$ & $71 / 24$ \\
\hline & & $K^{+}$ & $0.200 \pm 0.002$ & $0.200 \pm 0.003$ & $0.43 \pm 0.02$ & $24 / 13$ \\
\hline & & $p$ & $0.145 \pm 0.002$ & $0.326 \pm 0.002$ & $0.17 \pm 0.01$ & $120 / 24$ \\
\hline & & $\pi^{-}$ & $0.125 \pm 0.002$ & $0.383 \pm 0.002$ & $3.96 \pm 0.49$ & $54 / 24$ \\
\hline & & $K^{-}$ & $0.197 \pm 0.003$ & $0.190 \pm 0.003$ & $0.44 \pm 0.04$ & $14 / 13$ \\
\hline & & $\bar{p}$ & $0.144 \pm 0.001$ & $0.322 \pm 0.001$ & $0.13 \pm 0.01$ & $232 / 30$ \\
\hline & & $\phi$ & $0.112 \pm 0.002$ & $0.380 \pm 0.002$ & $0.0031 \pm 0.0003$ & $31 / 10$ \\
\hline & & $\Xi^{-}$ & $0.180 \pm 0.002$ & $0.298 \pm 0.005$ & $0.00042 \pm 0.00005$ & $12 / 8$ \\
\hline \multirow{10}{*}{$\begin{array}{c}\text { Fig. } 4 \\
\mathrm{~Pb}-\mathrm{Pb} \\
2.76 \mathrm{TeV}\end{array}$} & $0-5 \%$ & $\pi^{+}+\pi^{-}$ & $0.135 \pm 0.003$ & $0.432 \pm 0.003$ & $251.00 \pm 20.00$ & $5 / 38$ \\
\hline & & $K^{+}+K^{-}$ & $0.289 \pm 0.003$ & $0.264 \pm 0.002$ & $33.64 \pm 1.00$ & $4 / 33$ \\
\hline & & $p+\bar{p}$ & $0.443 \pm 0.002$ & $0.098 \pm 0.003$ & $10.50 \pm 0.50$ & $6 / 34$ \\
\hline & $0-10 \%$ & $\phi$ & $0.180 \pm 0.002$ & $0.370 \pm 0.002$ & $24.39 \pm 2.00$ & $1 / 5$ \\
\hline & & $\Xi^{-}$ & $0.320 \pm 0.002$ & $0.305 \pm 0.002$ & $3.80 \pm 0.03$ & $252 / 19$ \\
\hline & $70-80 \%$ & $\pi^{+}+\pi^{-}$ & $0.133 \pm 0.002$ & $0.420 \pm 0.002$ & $5.50 \pm 0.30$ & $10 / 38$ \\
\hline & & $K^{+}+K^{-}$ & $0.210 \pm 0.002$ & $0.347 \pm 0.002$ & $0.77 \pm 0.03$ & $2 / 33$ \\
\hline & & $p+\bar{p}$ & $0.222 \pm 0.001$ & $0.355 \pm 0.001$ & $0.27 \pm 0.01$ & $16 / 34$ \\
\hline & $60-80 \%$ & $\phi$ & $0.199 \pm 0.007$ & $0.405 \pm 0.007$ & $0.37 \pm 0.02$ & $5 / 5$ \\
\hline & & $\Xi^{-}$ & $0.183 \pm 0.009$ & $0.382 \pm 0.002$ & $0.13 \pm 0.02$ & $13 / 17$ \\
\hline \multirow{10}{*}{$\begin{array}{c}\text { Fig. } 5 \\
p-\mathrm{Pb} \\
5.02 \mathrm{TeV}\end{array}$} & $0-5 \%$ & $\pi^{+}+\pi^{-}$ & $0.119 \pm 0.001$ & $0.469 \pm 0.001$ & $7.23 \pm 0.04$ & $45 / 38$ \\
\hline & & $K^{+}+K^{-}$ & $0.293 \pm 0.003$ & $0.313 \pm 0.004$ & $0.98 \pm 0.04$ & $13 / 28$ \\
\hline & & $p+\bar{p}$ & $0.265 \pm 0.002$ & $0.393 \pm 0.002$ & $0.34 \pm 0.01$ & $12 / 36$ \\
\hline & & $\phi$ & $0.208 \pm 0.002$ & $0.435 \pm 0.001$ & $0.17 \pm 0.01$ & $16 / 11$ \\
\hline & & $\left(\Xi^{-}+\bar{\Xi}^{+}\right) / 2$ & $0.300 \pm 0.007$ & $0.390 \pm 0.005$ & $0.0099 \pm 0.0005$ & $16 / 12$ \\
\hline & $60-80 \%$ & $\pi^{+}+\pi^{-}$ & $0.120 \pm 0.003$ & $0.453 \pm 0.003$ & $1.45 \pm 0.10$ & $69 / 36$ \\
\hline & & $K^{+}+K^{-}$ & $0.232 \pm 0.002$ & $0.329 \pm 0.004$ & $0.19 \pm 0.01$ & $23 / 28$ \\
\hline & & $p+\bar{p}$ & $0.215 \pm 0.002$ & $0.365 \pm 0.002$ & $0.10 \pm 0.01$ & $107 / 36$ \\
\hline & & $\phi$ & $0.170 \pm 0.003$ & $0.440 \pm 0.002$ & $0.033 \pm 0.001$ & $15 / 11$ \\
\hline & & $\left(\Xi^{-}+\bar{\Xi}^{+}\right) / 2$ & $0.230 \pm 0.001$ & $0.399 \pm 0.002$ & $0.0017 \pm 0.0001$ & $4 / 12$ \\
\hline \multirow{5}{*}{$\begin{array}{c}\text { Fig. } 6 \\
p p \\
7 \mathrm{TeV}\end{array}$} & - & $\pi^{+}+\pi^{-}$ & $0.130 \pm 0.002$ & $0.430 \pm 0.002$ & $4.35 \pm 0.10$ & $206 / 35$ \\
\hline & & $K^{+}+K^{-}$ & $0.120 \pm 0.003$ & $0.458 \pm 0.002$ & $0.58 \pm 0.02$ & $374 / 43$ \\
\hline & & $p+\bar{p}$ & $0.204 \pm 0.003$ & $0.350 \pm 0.004$ & $0.25 \pm 0.01$ & $678 / 39$ \\
\hline & & $\phi$ & $0.130 \pm 0.002$ & $0.430 \pm 0.001$ & $0.031 \pm 0.003$ & $108 / 22$ \\
\hline & & $\Xi^{-}$ & $0.280 \pm 0.004$ & $0.320 \pm 0.005$ & $0.085 \pm 0.003$ & $138 / 18$ \\
\hline
\end{tabular}

imental data measured by the PHENIX Collaboration [11-13], where the spectra of neutral $\eta$ mesons and $\phi$ are cut at $5 \mathrm{GeV} / c$ due to a wide range being unnecessary for the extractions of $T_{0}$ and $\beta_{T}$. The left and right panels are the results corresponding to central (0-20\% cen- trality) and peripheral (60-88\% centrality) collisions respectively. The upper, middle, and lower panels are the results corresponding to $\pi^{+}, K^{+}$, and $p ; \pi^{-}, K^{-}$, and $\bar{p}$; as well as $\eta$ and $\phi$, respectively. Figure 3 is also the same as Fig. 1, but it shows the transverse momentum spectra 
with different expression in mid-rapidity interval in $p p$ collisions at center-of-mass energy $\sqrt{s}=200 \mathrm{GeV}$, where $E, \sigma$, and $N_{\mathrm{ev}}$ on the vertical axis denote the energy, cross section, and event number, respectively. The symbols represent the experimental data measured by the PHENIX [14] and STAR [17, 18] Collaborations. The left-upper, right-upper, and lower panels are the results corresponding to $\pi^{+}, K^{+}$, and $p$ in INEL events; $\pi^{-}$, $K^{-}$, and $\bar{p}$ in INEL events; as well as $\phi$ and $\Xi^{-}$in NSD events, respectively. One can see that the model results describe approximately the experimental data in special transverse momentum ranges in small collision system measured at the RHIC by the PHENIX and STAR Collaborations. The special transverse momentum ranges for some particles in peripheral $d$-Au collisions are only in $0-2 \sim 3 \mathrm{GeV} / c$ or a little more. In other cases, the particles are expected to have wider special transverse momentum ranges.

The transverse momentum spectra of different types of particles produced in mid-rapidity interval in $\mathrm{Pb}-\mathrm{Pb}$ collisions at $\sqrt{s_{N N}}=2.76 \mathrm{TeV}$ at the LHC are displayed in Fig. 4, where different expressions of $\phi$ and $\Xi^{-}$spectra are used, and different amounts marked in the panel are used to scale the spectra of $\pi^{+}+\pi^{-}$and $K^{+}+K^{-}$. The symbols represent the experimental data measured by the ALICE Collaboration [19-22], where the spectrum of $\Xi^{-}$is cut at $5 \mathrm{GeV} / c$. The curves are the results fitted by us using the blast-wave model with BoltzmannGibbs statistics, Eq. (1). Following each panel, the results of data/fit are presented. The left and right panels are the results corresponding to central $(0-5 \%$ or $0-10 \%$ centrality) and peripheral $(70-80 \%$ or $60-80 \%$ centrality) collisions respectively. The upper and lower panels are the results corresponding to $\pi^{+}+\pi^{-}, K^{+}+K^{-}$, and $p+\bar{p}$; as well as $\phi$ and $\Xi^{-}$, respectively. The values of $T_{0}, \beta_{T}, N_{0}, \chi^{2}$, and dof are listed in Table 1 . One can see that the model results describe approximately the experimental data in special transverse momentum ranges in large collision system measured at the LHC by the ALICE Collaboration. The special transverse momentum ranges in $\mathrm{Pb}-\mathrm{Pb}$ collisions are not observed in the available data ranges.

Figure 5 is the same as Fig. 4, but it shows the transverse momentum spectra of different types of particles produced in mid-rapidity interval in $p$ - $\mathrm{Pb}$ collisions at $\sqrt{s_{N N}}=5.02 \mathrm{TeV}$. The symbols represent the experimental data measured by the ALICE Collaboration [23-25], where the spectra of $\phi$ and $\left(\Xi^{-}+\bar{\Xi}^{+}\right) / 2$ are cut at $5 \mathrm{GeV} / c$, where $\bar{\Xi}^{+}$is anti-particle of positive $\Xi^{+}$baryon. The left and right panels are the results corresponding to central ( $0-5 \%$ centrality) and peripheral (60-80\% centrality) collisions respectively. The upper and lower panels are the results corresponding to $\pi^{+}+\pi^{-}, K^{+}+K^{-}$, and $p+\bar{p}$; as well as $\phi$ and $\left(\Xi^{-}+\bar{\Xi}^{+}\right) / 2$, respectively. Figure 6 is the same as Fig.
4, too, but it showing the transverse momentum spectra with different expression in mid-rapidity interval in $p p$ collisions at $\sqrt{s}=7 \mathrm{TeV}$, where $N_{\text {INEL }}$ and $N_{\text {NSD }}$ on the vertical axis denote the numbers of INEL and NSD events, respectively. The symbols represent the experimental data measured by the ALICE [26, 27] and CMS [28] Collaborations, where the spectra of $K^{+}+K^{-}, p+\bar{p}$, $\phi$, and $\Xi^{-}$are cut at $5 \mathrm{GeV} / c$. The left and right panels are the results corresponding to $\pi^{+}+\pi^{-}, K^{+}+K^{-}$, and $p+\bar{p}$; as well as $\phi$ and $\Xi^{-}$, respectively. One can see that the model results approximately consist with the experimental data in special transverse momentum ranges in small collision system measured at the LHC by the ALICE and CMS Collaborations. The special transverse momentum ranges in $p$ - $\mathrm{Pb}$ collisions are not obviously observed in the available data ranges. In $p p$ collisions, some particles are expected to have a special transverse momentum range in $0-3.5 \mathrm{GeV} / c$ or a little more.

To study the change trends of free parameters with rest mass of particle, Figure 7 gives the dependence of $T_{0}$ on $m_{0}$ (upper panel) and the dependence of $\beta_{T}$ on $m_{0}$ (lower panel). The left panels are for central and peripheral $\mathrm{Au}-\mathrm{Au}$ collisions, central and peripheral $d-\mathrm{Au}$ collisions, and $p p$ collisions at $200 \mathrm{GeV}$. The right panels are for central and peripheral $\mathrm{Pb}-\mathrm{Pb}$ collisions at 2.76 $\mathrm{TeV}$, central and peripheral $p$-Pb collisions at $5.02 \mathrm{TeV}$, and $p p$ collisions at $7 \mathrm{TeV}$. Different symbols represent values of parameters in different collisions from Table 1. One can see that $T_{0}\left(\beta_{T}\right)$ increases (decreases) slightly with the increase of $m_{0} . T_{0}\left(\beta_{T}\right)$ in central collisions is slightly larger than or nearly equal to that in peripheral collisions. $T_{0}\left(\beta_{T}\right)$ in collisions at the LHC is slightly larger than or nearly equal to that in collisions at the RHIC. $p p$ collisions are closer to peripheral nuclear collisions.

The fact that $T_{0}$ is dependent of $m_{0}$ reveals the scenario for multiple kinetic freeze-out at the RHIC and LHC $[35,56,57]$. The scenario for single or double kinetic freeze-out $[29,58,59]$ is not observed in this study. Just as its name implies, the scenario for multiple kinetic freeze-out uses different sets of parameters for the spectra of different particles with different masses, i.e., this scenario is the mass-dependent and differential. The scenario for single kinetic freeze-out uses one set of parameters for the spectra of all particles which includes both the strange and non-strange particles. The scenario for double kinetic freeze-out uses different sets of parameters for strange and non-strange particles. Although the average values of parameters weighted by different particles yield in multiple kinetic freeze-out scenario can be regarded as the parameters in single kinetic freeze-out scenario, the fit results will be unacceptable due to large departure from the data.

In fact, if we use average $T_{0}$ and $\beta_{T}$ as the parame- 

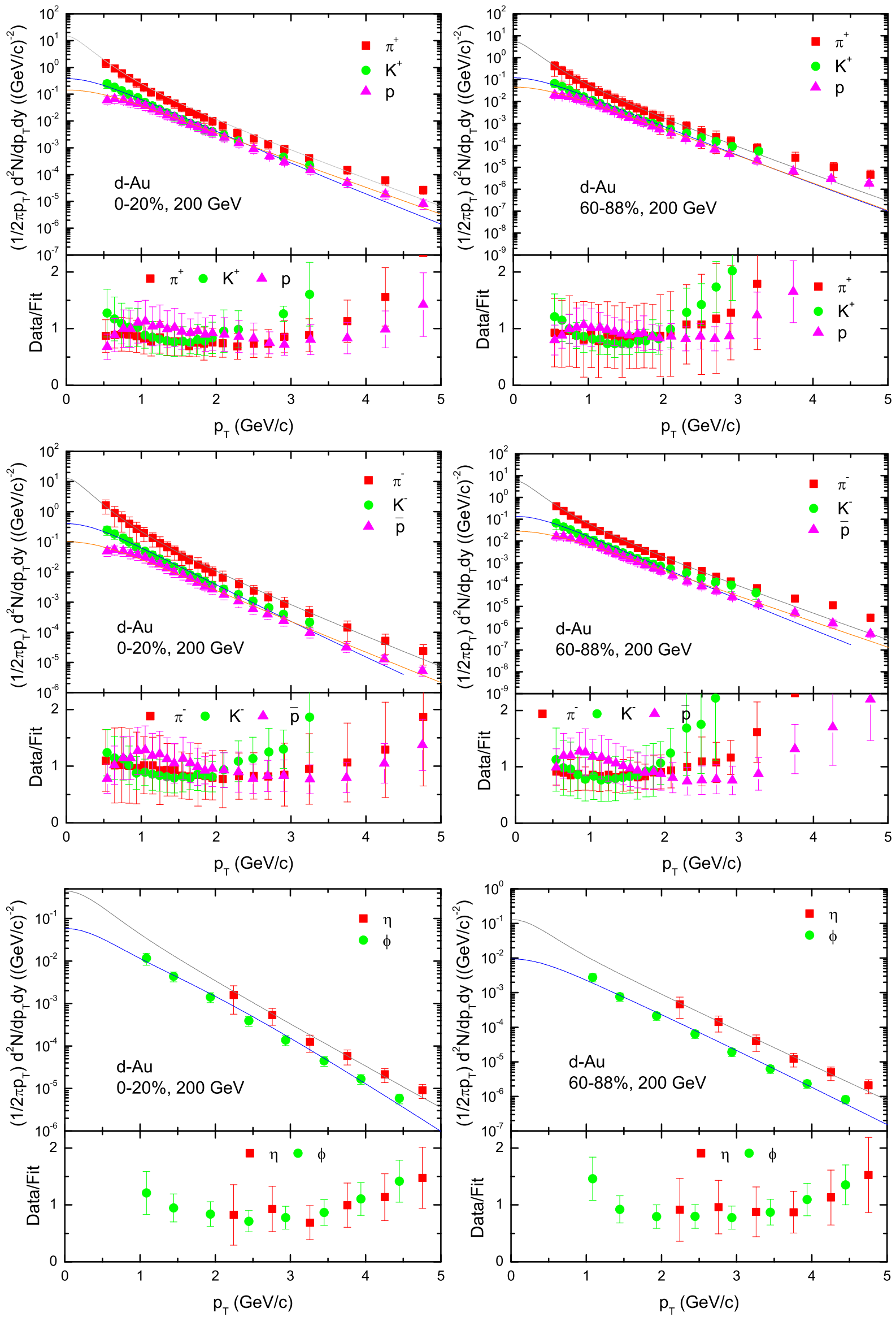

Fig. 2. Same as Fig. 1, but showing the spectra in $d$-Au collisions at $\sqrt{s_{N N}}=200 \mathrm{GeV}$. The symbols represent the experimental data measured by the PHENIX Collaboration [11-13], where the spectra of $\eta$ and $\phi$ are cut at 5 $\mathrm{GeV} / c$ due to a wide range being unnecessary for the extractions of $T_{0}$ and $\beta_{T}$. Left: for central collisions $(0-20 \%$ centrality); Right: for peripheral collisions (60-88\% centrality). Upper: for $\pi^{+}, K^{+}$, and $p$ with $|\eta|<0.35$ [11]; Middle: for $\pi^{-}, K^{-}$, and $\bar{p}$ with $|\eta|<0.35$ [11]; Lower: for $\eta[12]$ and $\phi$ [13] with $|\eta|<0.35$. 

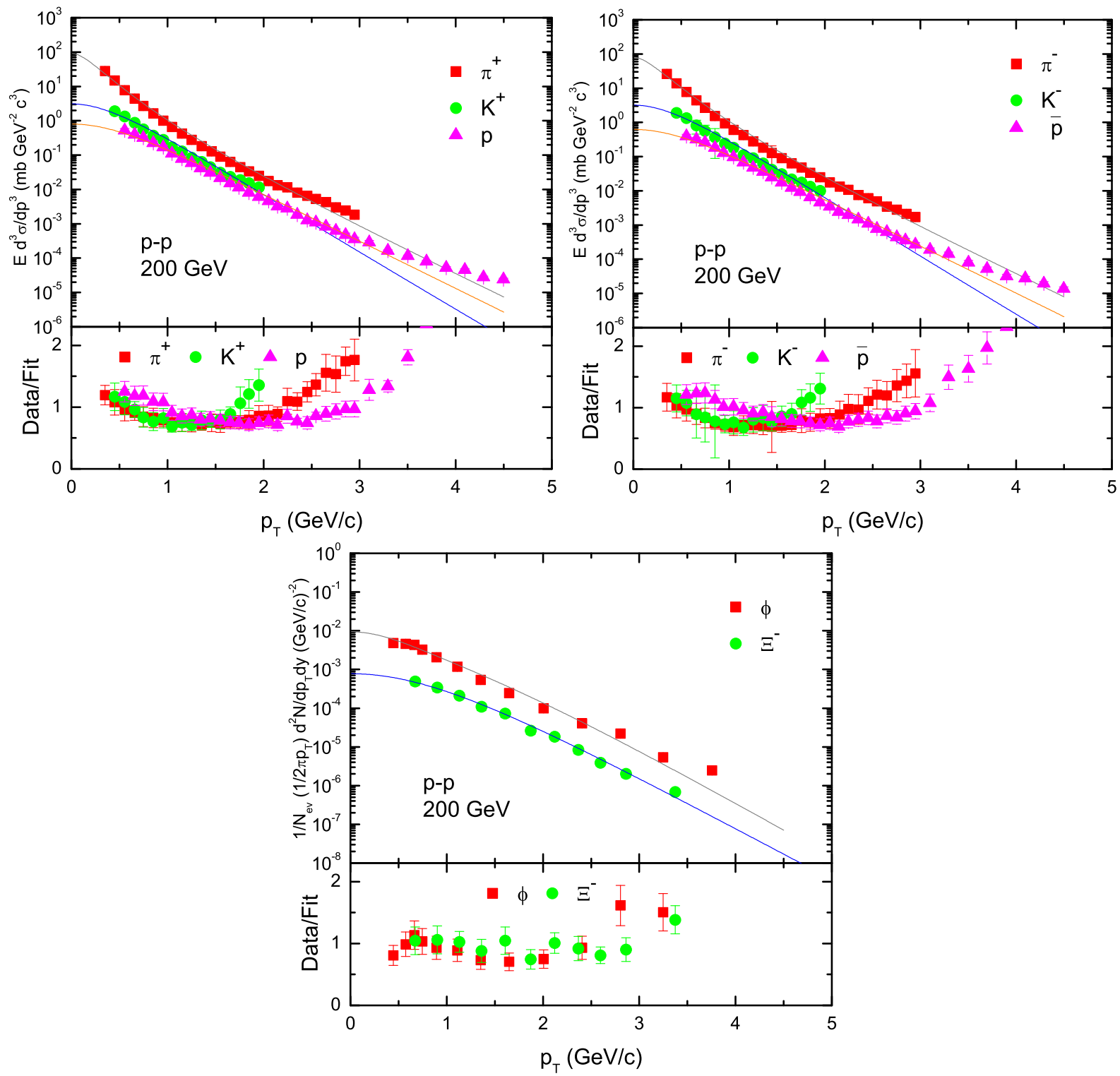

Fig. 3. Same as Fig. 1, but showing the spectra with different expression in $p p$ collisions at $\sqrt{s}=200 \mathrm{GeV}$, where $E, \sigma$, and $N_{\mathrm{ev}}$ denote the energy, cross section, and event number, respectively, and $N_{\mathrm{ev}}$ is usually omitted. The symbols represent the experimental data measured by the PHENIX [14] and STAR [17, 18] Collaborations in INEL and NSD events respectively. Upper: for $\pi^{+}, K^{+}$, and $p$ (left), as well as $\pi^{-}, K^{-}$, and $\bar{p}$ (right) with $|\eta|<0.35$ in INEL events [14]; Lower: for $\phi[17]$ and $\Xi^{-}[18]$ with $|y|<0.5$ in NSD events.

ters for single freeze-out scenario, the fitting results for pion spectra are approximately acceptable due to these parameters being close to those for pion emission. The fitting results for kaon and proton spectra are not acceptable due to very large $\chi^{2}$ (in some cases $\chi^{2}>1000$ ). In the case of double freeze-out scenario, the average parameters weighted from the pion and proton spectra can fit approximately the pion spectra, and cannot fit the proton spectra, though the kaon spectra are described by another set of parameters. Even if we fit the spectra in a narrower $p_{T}$ range, the fitting results by the single and double freeze-out scenarios are not acceptable. In view of this situation, the present work does not support the single and double freeze-out scenarios, but the multiple kinetic freeze-out scenario.

The relative sizes of $T_{0}\left(\beta_{T}\right)$ in central and peripheral nuclear collisions, as well as in collisions at the RHIC and LHC are harmonious to our recent works $[5,60]$ in general, though the contribution of soft excitation is included and the contribution of hard scattering processes is excluded in low $p_{T}$ region in [5]. Oppositely, the present work includes the contributions of soft ex- 

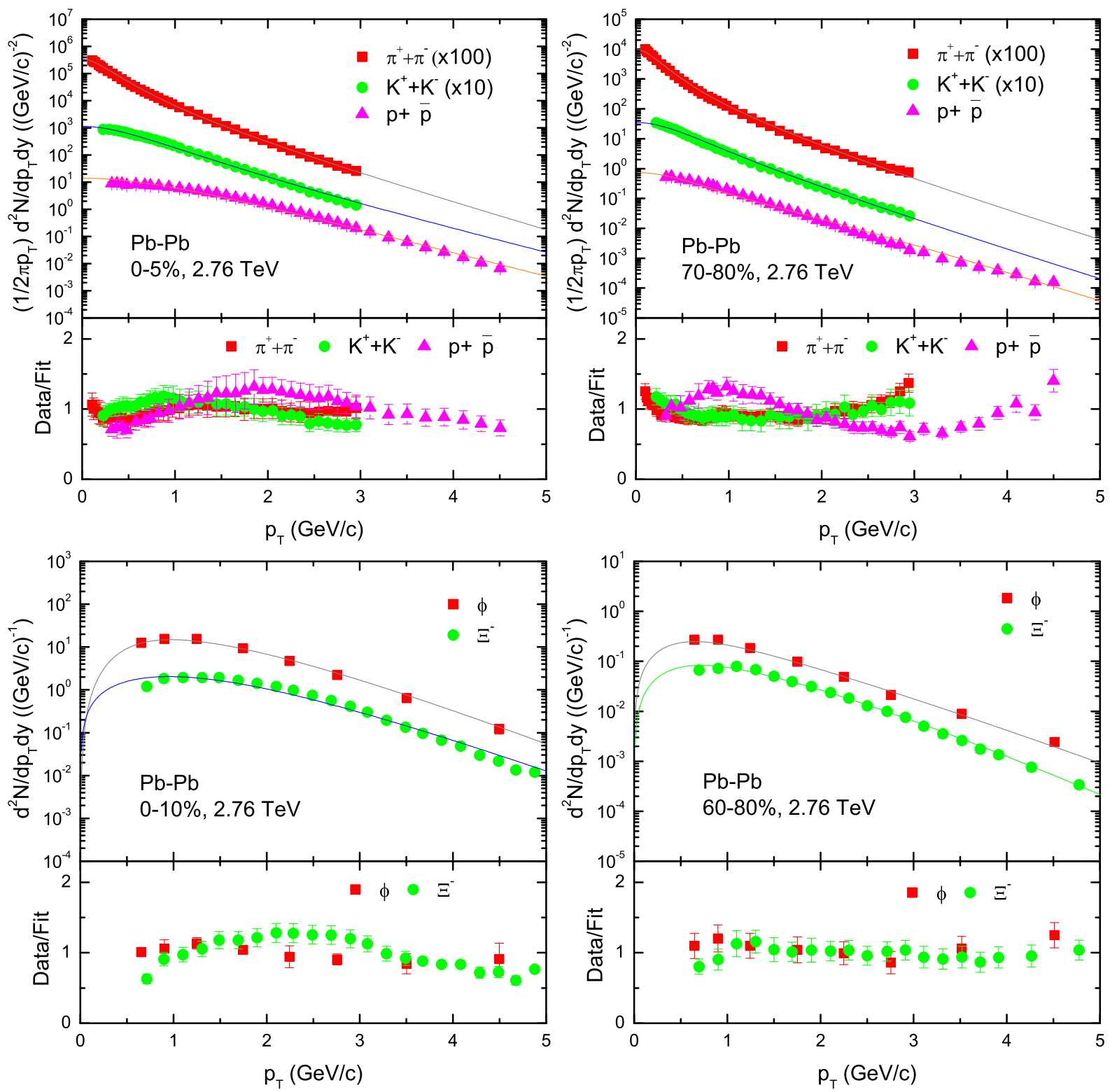

Fig. 4. Transverse momentum spectra of different types of particles produced in $\mathrm{Pb}-\mathrm{Pb}$ collisions at $\sqrt{s_{N N}}=2.76$ $\mathrm{TeV}$ at the LHC, where different expression of $\phi$ and $\Xi^{-}$spectra is used. The symbols represent the experimental data measured by the ALICE Collaboration [19-22], where the spectrum of $\Xi^{-}$is cut at $5 \mathrm{GeV} / c$. The curves are the results fitted by us using the blast-wave model with Boltzmann-Gibbs statistics, Eq. (1). Left: for central collisions ( $0-5 \%$ or $0-10 \%$ centrality); Right: for peripheral collisions ( $70-80 \%$ or $60-80 \%$ centrality). Upper: for $\pi^{+}+\pi^{-}, K^{+}+K^{-}$, and $p+\bar{p}$ with $|y|<0.5$, where different amounts marked in the panel are used to scale the spectra [19, 20]; Lower: for $\phi$ with $|y|<0.5$ [21] and $\Xi^{-}$with $|y|<0.5$ at $p_{T}>1.8 \mathrm{GeV} / c$ and with $|y|<0.3$ at $p_{T}<1.8 \mathrm{GeV} / c[22]$.

citation process and hard scattering process in low $p_{T}$ region [60] in the Hagedorn's model [Eq. (4)], if the contribution of hard process in low $p_{T}$ region is available. This causes that there are some small differences in absolute values from our previous work [5]. More discussions on the relative sizes of $T_{0}\left(\beta_{T}\right)$ and comparisons with other works can be found in [60].
Although this work presents two superpositions, i.e. Eqs. (3) and (4), they are not directly used to fit the data in Figs. 1-6. Instead, Eq. (1) is simply used to fit the mentioned data. This treatment uses in fact only the first component in Eqs. (3) and (4), and it is more close to Eq. (4). In Eq. (4), the contribution of hard process in low $p_{T}$ region is not excluded, though it does 

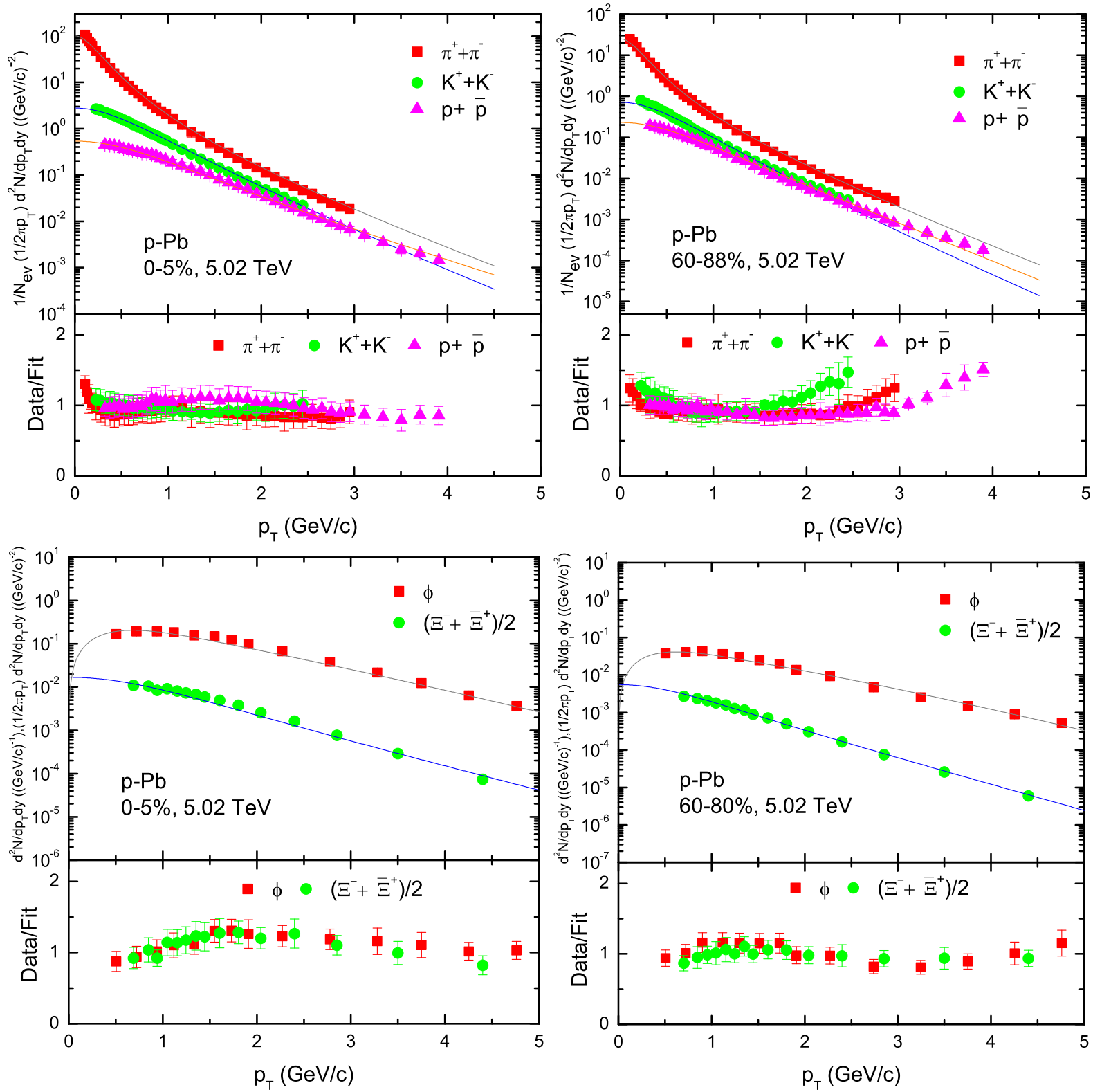

Fig. 5. Same as Fig. 4, but showing the spectra in $p$ - $\mathrm{Pb}$ collisions at $\sqrt{s_{N N}}=5.02 \mathrm{TeV}$, where different expression of $\phi$ spectra is used. The symbols represent the experimental data measured by the ALICE Collaboration [23-25], where the spectra of $\phi$ and $\left(\Xi^{-}+\bar{\Xi}^{+}\right) / 2$ are cut at $5 \mathrm{GeV} / c$. Left: for central collisions $(0-5 \%$ centrality); Right: for peripheral collisions (70-80\% centrality). Upper: for $\pi^{+}+\pi^{-}, K^{+}+K^{-}$, and $p+\bar{p}$ with $0<y<0.5$ [23]; Lower: for $\phi[24]$ and $\left(\Xi^{-}+\bar{\Xi}^{+}\right) / 2[25]$ with $-0.5<y<0$.

not get entanglement in the determination of parameters of the two components. While, Eq. (3) includes the contribution of hard process in low $p_{T}$ region, and it gets entanglement in the determination of parameters. To show main topic, the present work gives up to use Eqs. (3) and (4) in high $p_{T}$ region beyond $5 \mathrm{GeV} / c$, though most data are only in low $p_{T}$ region. Instead, Eqs. (3) and (4) are used in our recent works [5] and [60] respectively, in which the functional trends in high $p_{T}$ region can be clearly seen.
In our opinion, Eqs. (3) and (4) can be used to quantify the centrality, rapidity, and energy dependent soft versus hard contributions to particle production. This would bring some new idea to business. For example, from the comparisons between the curves and data in Figs. 1-3, we can see that the fraction of hard contribution in peripheral collisions is greater than that in central collisions. The fraction of hard contribution in $p p$ collisions is greater than that in central $d$-Au collisions, and the latter is greater than that in central 

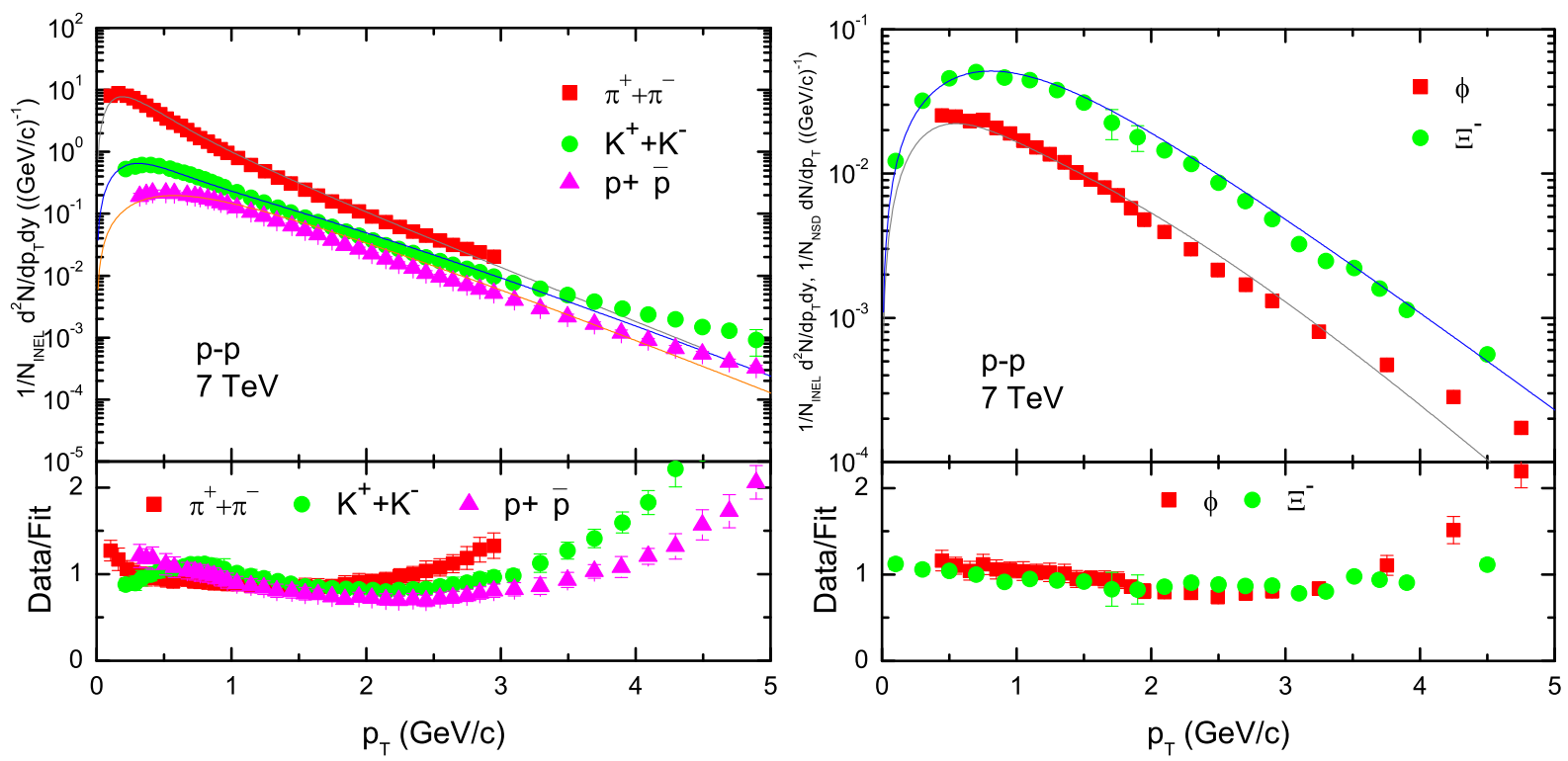

Fig. 6. Same as Fig. 4, but showing the spectra with different expressions in $p p$ collisions at $\sqrt{s}=7 \mathrm{TeV}$, where $N_{\text {INEL }}$ and $N_{\text {NSD }}$ denote the numbers of INEL and NSD events respectively. The symbols represent the experimental data measured by the ALICE $[26,27]$ and CMS [28] Collaborations, where the spectra of $K^{+}+K^{-}, p+\bar{p}$, $\phi$, and $\Xi^{-}$are cut at $5 \mathrm{GeV} / c$. Left: for $\pi^{+}+\pi^{-}, K^{+}+K^{-}$, and $p+\bar{p}$ with $|y|<0.5$ in INEL events [26]; Right: for $\phi$ with $|y|<0.5$ in INEL events [27] and $\Xi^{-}$with $|y|<2$ in NSD events [28].

$\mathrm{Au}-\mathrm{Au}$ collisions. The reason is less cascade or intranuclear collisions in peripheral collisions and in small system. Generally, the fractions in these collisions are about a few percentages to above ten percentages [5, 60]. To make a definitive conclusion on other dependences, more and systematic data are needed. This is beyond the focus of the present work. We shall not discuss them anymore.

The values of average $T_{0}\left(\left\langle T_{0}\right\rangle\right)$ and average $\beta_{T}$ $\left(\left\langle\beta_{T}\right\rangle\right)$ for different types of collisions at the RHIC and LHC are listed in Table 2 . These average values are obtained by different weights due to different yields $\left(N_{0}\right)$ of $\pi^{+}+\pi^{-}, K^{+}+K^{-}$, and $p+\bar{p}$. Other particles are not included in the averages due to non-identity type and centrality. In particular, $\left\langle T_{0}\right\rangle$ in central nuclear collisions is $\sim 148 \mathrm{MeV}$ which is less than $T_{c h}(\sim 160 \mathrm{MeV}$ [1-4]), which renders that the kinetic freeze-out in central collisions at the considered energies happens later than the chemical freeze-out by $\sim 2 \mathrm{fm}$ according to the time evolution of temperature, $T_{f}=T_{i}\left(\tau_{i} / \tau_{f}\right)^{1 / 3}[61$, $62]$, where $T_{i}(=300 \mathrm{MeV})$ and $\tau_{i}(=1 \mathrm{fm})$ are the initial temperature and proper time respectively [62]. Eq. (4) used in our recent work [60] results in larger $T_{0}$ and $\beta_{T}$ than Eq. (3) used in our another work [5] due to the non-exclusion of hard process in Eq. (4).

In different types of collisions, the values of $T_{0}$ and $\beta_{T}$ for emissions of pions show small fluctuations due to large yields and high statistics. The values of $T_{0}$ and $\beta_{T}$ for emissions of other particles show large fluctua- tions due to small yields and low statistics. Because of large yields of pions, $\left\langle T_{0}\right\rangle$ and $\left\langle\beta_{0}\right\rangle$ weighted for yields of different particles are closer to those for emissions of poins. Since the emissions of other particles require higher $T_{0}$, other particles freeze-out earlier than pions at the kinetic freeze-out stage. To study accurately dependences of $T_{0}\left(\beta_{T}\right)$ on centrality and energy, one can select the spectra of pions, at the most including the spectra of kaons and (anti-)protons. In fact, in the alternative method discussed in the beginning of section 2 , the spectra of the three types of particles are needed.

Note that the extracted mass dependent $T_{0}$ and $\beta_{T}$ in central collisions are comparable with those in peripheral collisions. In particular, the behavior of $\beta_{T}$ is different from that of the elliptic flow $v_{2}$ because $\beta_{T}$ describes the flow velocity in the transverse plane and $v_{2}$ describes the flow anisotropy in the transverse plane. In central collisions, $v_{2}$ is approximately equal to 0 due to the approximately isotropy. While in peripheral collisions, $v_{2}$ is considerable due to the anisotropy. In some cases, the velocity may be small, and the anisotropy may be large. The mass dependent parameters may be related to the scaling of the number of constituent quarks. The concrete relation is worth to be studied in the future.

We would like to point out that the errors of parameters are very small due to strict restrictions $\left(\chi_{\max }^{2} \leq\right.$ $1.05 \chi_{\min }^{2}$ ) being used in the fits (where $\chi_{\max }^{2}$ denotes the maximum- $\chi^{2}$ when we determine the errors and $\chi_{\min }^{2}$ 

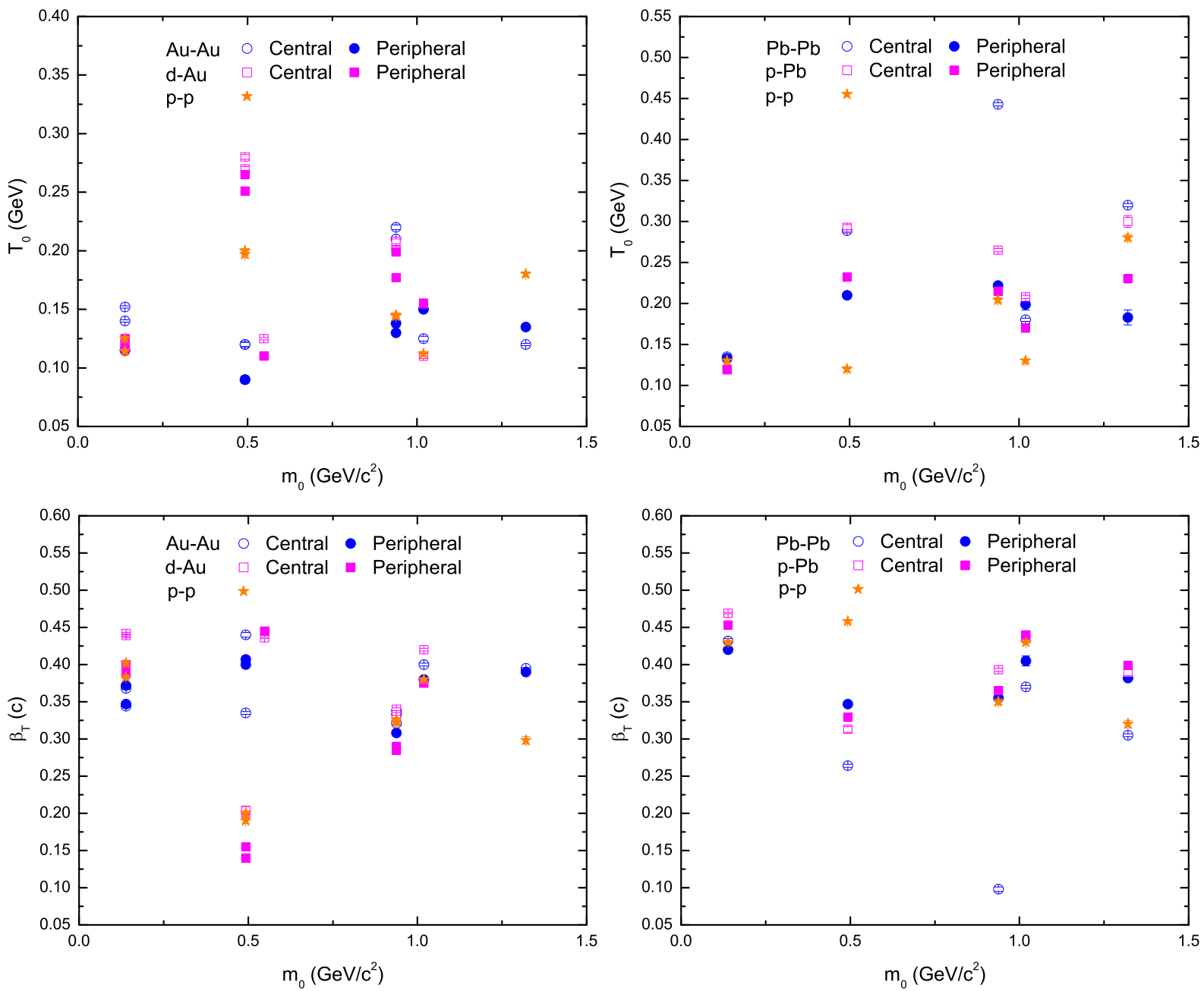

Fig. 7. Upper: dependence of $T_{0}$ on $m_{0}$; Lower: dependence of $\beta_{T}$ on $m_{0}$. Left: for central and peripheral Au-Au collisions, central and peripheral $d$-Au collisions, and INEL or NSD $p p$ collisions at $200 \mathrm{GeV}$; Right: for central and peripheral $\mathrm{Pb}-\mathrm{Pb}$ collisions at $2.76 \mathrm{TeV}$, central and peripheral $p$ - $\mathrm{Pb}$ collisions at $5.02 \mathrm{TeV}$, and INEL or NSD $p p$ collisions at $7 \mathrm{TeV}$. Different symbols represent values of parameters in different collisions, which are taken from Table 1.

denotes the minimum- $\chi^{2}$ when we determine the best parameters). If we use weak restrictions, large errors will be obtained. However, in this case, large $\chi^{2}$ will also be obtained, which shows bad fitting results. On the other hand, as a fitting function itself, Eq. (4) is not ideal due to fewer parameters being used in low $p_{T}$ region, which renders small variable ranges of parameters in limited selection. Instead, Eq. (3) can give better fit due to more parameters being used in low $p_{T}$ region, which renders large variable ranges of parameters by flexible selection. Indeed, the limited selection restricts $T_{0}$ and $\beta_{T}$ themselves.

Before the final conclusions, it should be emphasized that the successful fitting of the blast-wave model for the experimental data studied reveals that the properties of interacting system and produced particles at the kinetic stage is abundant. The excitation degree of interacting system and the collective motion of produced particles are described in the model by $T_{0}$ and $\beta_{T}$ respectively. According to $T_{0}$ and $\beta_{T}$ for the emissions of different particles, we can study various possible scenarios which includes single, double, or multiple kinetic freeze-out. The present work conforms the multiple kinetic freezeout scenario. Although the blast-wave model is not the only option in the extraction of $T_{0}$ and $\beta_{T}$, it is one of the applicable methods.

In particular, the blast-wave model makes an assumption that particles are locally thermalized at a kinetic freeze-out temperature and are moving with a common collective transverse radial flow velocity field. It seems that a single freeze-out scenario, that is the same $T_{0}$ and $\beta_{T}$, should be taken into consideration for different particles. The present work shows that $T_{0}$ and $\beta_{T}$ for the emissions of different particles are severally 
Table 2. Values of $\left\langle T_{0}\right\rangle$ and $\left\langle\beta_{T}\right\rangle$ in different types of collisions at the RHIC and LHC. The average values are obtained by different weights due to different yields $\left(N_{0}\right)$ of $\pi^{+}+\pi^{-}, K^{+}+K^{-}$, and $p+\bar{p}$. Other particles are not included in the averages due to non-identity type and centrality.

\begin{tabular}{cccc}
\hline \hline Collisions & Energy & $\left\langle T_{0}\right\rangle(\mathrm{GeV})$ & $\left\langle\beta_{T}\right\rangle(c)$ \\
\hline Central $\mathrm{Au}-\mathrm{Au}$ & $200 \mathrm{GeV}$ & $0.145 \pm 0.009$ & $0.366 \pm 0.018$ \\
Peripheral $\mathrm{Au}-\mathrm{Au}$ & $200 \mathrm{GeV}$ & $0.113 \pm 0.006$ & $0.363 \pm 0.013$ \\
Central $d-\mathrm{Au}$ & $200 \mathrm{GeV}$ & $0.140 \pm 0.013$ & $0.411 \pm 0.045$ \\
Peripheral $d-\mathrm{Au}$ & $200 \mathrm{GeV}$ & $0.135 \pm 0.013$ & $0.369 \pm 0.041$ \\
INEL or NSD $p p$ & $200 \mathrm{GeV}$ & $0.128 \pm 0.006$ & $0.371 \pm 0.017$ \\
\hline Central Pb-Pb & $2.76 \mathrm{TeV}$ & $0.164 \pm 0.014$ & $0.410 \pm 0.037$ \\
Peripheral Pb-Pb & $2.76 \mathrm{TeV}$ & $0.146 \pm 0.009$ & $0.409 \pm 0.025$ \\
Central $p-\mathrm{Pb}$ & $5.02 \mathrm{TeV}$ & $0.145 \pm 0.002$ & $0.448 \pm 0.004$ \\
Peripheral $p-\mathrm{Pb}$ & $5.02 \mathrm{TeV}$ & $0.137 \pm 0.011$ & $0.435 \pm 0.033$ \\
INEL or NSD $p p$ & $7 \mathrm{TeV}$ & $0.133 \pm 0.005$ & $0.429 \pm 0.012$ \\
\hline
\end{tabular}

different. This means that the interacting system is locally thermalzied or successionally emissive in various types of collisions. Even in peripheral nucleus-nucleus collisions and in $p p$ collisions, the multiplicity per unit rapidity at the mid-rapidity is enough high at the RHIC and LHC, and the concept of equilibrium statistical mechanics can be used.

\section{Conclusions}

We summarize here our main observations and conclusions.

(a) The transverse momentum spectra of different types of particles produced in mid-rapidity interval in central and peripheral $\mathrm{Au}-\mathrm{Au}$ collisions at $200 \mathrm{GeV}$, central and peripheral $d$-Au collisions at $200 \mathrm{GeV}$, and INEL or NSD $p p$ collisions at $200 \mathrm{GeV}$, as well as in central and peripheral $\mathrm{Pb}-\mathrm{Pb}$ collisions at $2.76 \mathrm{TeV}$, central and peripheral $p$ - $\mathrm{Pb}$ collisions at $5.02 \mathrm{TeV}$, and INEL or NSD $p p$ collisions at $7 \mathrm{TeV}$ are analyzed by the blastwave model with Boltzmann-Gibbs statistics. In the model fit, the contributions of soft excitation and hard scattering processes are included in low transverse momentum region. This treatment of Hagedorn's model leads to fewer parameters and smaller errors of parameters in low transverse momentum region. The model results are approximately in agreement with the experimental data measured at the RHIC by the PHENIX and STAR Collaborations and at the LHC by the ALICE and CMS Collaborations.

(b) The kinetic freeze-out temperature increases slightly and the transverse flow velocity decreases slightly with the increase of particle mass. The kinetic freeze-out temperature in central collisions is slightly larger than or nearly equal to that in peripheral collisions, and that in collisions at the LHC is slightly larger than or nearly equal to that in collisions at the RHIC. The dependences of transverse flow velocity on centrality and energy are similar to those of the kinetic freeze-out temperature. The similarity of $p p$ collisions to peripheral collisions is observed by the Hagedorn's model in which the contribution of hard process in low transverse momentum region is included. The fact that the kinetic freeze-out temperature is dependent on particle mass reveals the scenario for multiple kinetic freeze-out at the RHIC and LHC. The scenario for single or double kinetic freeze-out is not observed in this study.

\section{Data Availability}

The data used to support the findings of this study are quoted from the mentioned references. As a phenomenological work, this paper does not report new data.

\section{Conflicts of Interest}

The authors declare that there are no conflicts of interest regarding the publication of this paper.

\section{Acknowledgments}

Communications from Edward K. SarkisyanGrinbaum are highly acknowledged. This work was supported by the National Natural Science Foundation of China under Grant Nos. 11575103 and 11747319, the Chinese Government Scholarship (China Scholarship Council), the Shanxi Provincial Natural Science Foundation under Grant No. 201701D121005 (China), the Fund for Shanxi "1331 Project" Key Subjects Construction (China), and the Grant of Scientific Research Deanship at Qassim University (Kingdom of Saudi Arabia).

\section{References}

[1] A Andronic, P Braun-Munzinger and J Stachel Nucl. Phys. A772 167 (2006)

[2] J Cleymans, H Oeschler, K Redlich and S Wheaton Phys. Rev. C73 034905 (2006)

[3] A Andronic, P Braun-Munzinger, J Stachel Acta Phys. Pol. B40 1005 (2009) 
[4] A Andronic, P Braun-Munzinger and J Stachel Nucl. Phys. A834 237c (2010)

[5] H-L Lao, F-H Liu, B-C Li and M-Y Duan Nucl. Sci. Tech. 2982 (2018)

[6] H C Song, Y Zhou and K Gajdošová Nucl. Sci. Tech. 2899 (2017)

[7] E Schnedermann, J Sollfrank and U Heinz Phys. Rev. C48 2462 (1993)

[8] B I Abelev et al. [STAR Collaboration] Phys. Rev. C79 034909 (2009)

[9] B I Abelev et al. [STAR Collaboration] Phys. Rev. C81 024911 (2010)

[10] S S Adler et al. [PHENIX Collaboration] Phys. Rev. C69 034909 (2004)

[11] A Adare et al. [PHENIX Collaboration] Phys. Rev. C88 024906 (2013)

[12] S S Adler et al. [PHENIX Collaboration] Phys. Rev. C75 024909 (2007)

[13] A Adare et al. [PHENIX Collaboration] Phys. Rev. C83 024909 (2011)

[14] A Adare et al. [PHENIX Collaboration] Phys. Rev. C83 064903 (2011)

[15] B I Abelev et al. [STAR Collaboration] Phys. Rev. Lett. 99112301 (2007)

[16] J Adams et al. [STAR Collaboration] Phys. Rev. Lett. 98062301 (2007)

[17] J Adams et al. [STAR Collaboration] Phys. Lett. B612 $181(2005)$

[18] B I Abelev et al. [STAR Collaboration] Phys. Rev. C75 064901 (2007)

[19] B Abelev et al. [ALICE Collaboration] Phys. Rev. Lett. 109252301 (2012)

[20] B Abelev et al. [ALICE Collaboration] Phys. Rev. C88 044910 (2013)

[21] B Abelev et al. [ALICE Collaboration] Phys. Rev. C91 024609 (2015)

[22] B Abelev et al. [ALICE Collaboration] Phys. Lett. B728 216 (2014) and Erratum Phys. Lett. B734 409 (2014)

[23] B Abelev et al. [ALICE Collaboration] Phys. Lett. B728 25 (2014)

[24] J Adam et al. [ALICE Collaboration] Eur. Phys. J. C76 $245(2016)$

[25] J Adam et al. [ALICE Collaboration] Phys. Lett. B758 389 (2016)

[26] J Adam et al. [ALICE Collaboration] Eur. Phys. J. C75 $226(2015)$

[27] B Abelev et al. [ALICE Collaboration] Eur. Phys. J. C72 2183 (2012)

[28] V Khachatryan et al. [CMS Collaboration] JHEP 05 064 (2011)

[29] Z B Tang, Y C Xu, L J Ruan, G van Buren, F Q Wang and Z B Xu Phys. Rev. C79 051901(R) (2009)

[30] S Takeuchi, K Murase, T Hirano, P Huovinen and Y Nara Phys. Rev. C92 044907 (2015)

[31] H Heiselberg and A M Levy Phys. Rev. C59 2716 (1999)

[32] U W Heinz Lecture Notes for Lectures Presented at the 2nd CERN-Latin-American School of High-Energy Physics (1-14 June 2003, San Miguel Regla, Mexico), arXiv:hep-ph/0407360 (2004)
[33] R Russo PhD Thesis (Universita degli Studi di Torino, Italy) (2015), arXiv:1511.04380 [nucl-ex] (2015)

[34] H-R Wei, F-H Liu and R A Lacey Eur. Phys. J. A52 $102(2016)$

[35] H-L Lao, H-R Wei, F-H Liu and R A Lacey Eur. Phys. J. A52 203 (2016)

[36] H-R Wei, F-H Liu and R A Lacey J. Phys. G43 125102 (2016)

[37] J Cleymans and D Worku Eur. Phys. J. A48 160 (2012)

[38] H Zheng and L L Zhu Adv. High Energy Phys. 2016 9632126 (2016)

[39] S Zhang, Y G Ma, J H Chen and C Zhong Adv. High Energy Phys. 2015460590 (2015)

[40] A Bialas, W Florkowski and K Zalewski J. Phys. G42 045001 (2015)

[41] X Sun, H Masui, A M Poskanzer and A Schmah Phys. Rev. C91 024903 (2015)

[42] W Florkowski Acta Phys. Pol. B47 2241 (2016)

[43] J Cimerman, B Tomasik, M Csanad and S Lokos Eur. Phys. J. A53 161 (2017)

[44] R Odorico Phys. Lett. B118 151 (1982)

[45] G Arnison et al. [UA1 Collaboration] Phys. Lett. B118 167 (1982)

[46] T Mizoguchi, M Biyajima and N Suzuki Int. J. Mod. Phys. A32 1750057 (2017)

[47] R Hagedorn Riv. Nuovo Cimento 61 (1983)

[48] B Abelev et al. [ALICE Collaboration] Eur. Phys. J. C75 1 (2015)

[49] K Aamodt et al. [ALICE Collaboration] Phys. Lett. B693 53 (2010)

[50] A De Falco [for the ALICE Collaboration] J. Phys. G38 124083 (2011)

[51] I Abt et al. [HERA-B Collaboration] Eur. Phys. J. C50 315 (2007).

[52] B Abelev et al. [ALICE Collaboration] Phys. Lett. B710 557 (2012)

[53] B Abelev et al. [ALICE Collaboration] Phys. Lett. B718 295 (2012) and Erratum Phys. Lett. B748 472 (2015)

[54] I Lakomov [for the ALICE collaboration] Nucl. Phys. A931 1179 (2014)

[55] B Abelev et al. [ALICE Collaboration] Phys. Lett. B708 265 (2012)

[56] S Chatterjee and B Mohanty Phys. Rev. C90 034908 (2014)

[57] D Thakur, S Tripathy, P Garg, R Sahoo and J Cleymans Adv. High Energy Phys. 20164149352 (2016)

[58] S Chatterjee, S Das, L Kumar, D Mishra, B Mohanty, R Sahoo and N Sharma Adv. High Energy Phys. 2015 349013 (2015)

[59] S Chatterjee, B Mohanty and R Singh Phys. Rev. C92 024917 (2015)

[60] H-L Lao, F-H Liu, B-C Li, M-Y Duan and R A Lacey, Nucl. Sci. Tech. 29164 (2018)

[61] J D Bjorken Phys. Rev. D27 140 (1983)

[62] K Okamoto and C Nonaka Eur. Phys. J. C77 383 (2017) 